\title{
Osteitic bone: a surrogate marker of eosinophilia in chronic rhinosinusitis*
}

\author{
Kornkiat Snidvongs ${ }^{1}$, Rohan McLachlan², David Chin³, Eleanor Pratt ${ }^{4}$, \\ Raymond Sacks ${ }^{5}$, Peter Earls ${ }^{6}$, Richard J. Harvey ${ }^{7}$ \\ Rhinology 50: 299-305, 2012 \\ DOI: 10.4193/Rhino12.022 \\ Macquarie University, Australia and Faculty of Medicine, Chulalongkorn University, Thailand \\ *Received for publication: \\ University of New South Wales, Australia \\ January 27, 2012 \\ St Vincent's Hospital, Australia \\ Accepted: March 7, 2012 \\ University of Sydney, Australia \\ Macquarie University, University of Sydney, Australia \\ Department of anatomical pathology, St Vincent's Hospital, Australia \\ St Vincent's Hospital, Macquarie University and University of New South Wales, Australia
}

\section{Summary}

Background: Causes of osteitis in chronic rhinosinusitis (CRS) other than previous surgery are poorly defined. Patients with eosinophilic CRS (ECRS) have more severe disease and poorer outcomes despite repeated surgery. Associations between osteitis and markers of ECRS are not well described.

Methods: A cross-sectional study of CRS patients undergoing sinus surgery was conducted. Osteitis was scored radiologically using previously published measures. Associations between osteitis and histopathology, symptoms, endoscopy, CT mucosal score and seromarkers were analyzed.

Results: Eighty-eight patients were assessed of whom forty-five had osteitis. Patients undergoing revision surgery recorded higher osteitis scores. Patients with mucosal eosinophilia had higher osteitis score than those without. Patients with osteitis had higher serum eosinophil. Similar relationships were also found in primary surgery. Osteitis was associated with endoscopic and radiologic, but not symptomatic disease severity.

Conclusions: Osteitis is associated with tissue and serum eosinophilia in both patients with and without prior surgery. Patients with these features may benefit from post-operative corticosteroid therapy to prevent osteitis.

Key words: osteitis, eosinophilia, eosinophils, sinusitis, polyps

\section{Introduction}

Osteitic bone is a feature of chronic rhinosinusitis (CRS) and often associated with recalcitrant disease ${ }^{(1)}$. The osteitic bones potentially serve as a nidus for inflammation and may explain failures from typical medical and surgical treatment. Osteitis is associated with previous surgery and the incidence increases with increasing number of previous operations (2). However, non-operated patients also experience osteitis with an incidence of $5^{(3)}$ to $33 \%{ }^{(2)}$ and thus mucosal loss from surgery is not a simple answer to the origins and implication of osteitis (4). The mechanism of osteitis in CRS is poorly understood and yet to be fully defined. Although, bacteria may play a role in 
the pathogenesis by infecting sinus walls or producing biofilm which results in the release of mediators, to date bacteria have not been demonstrated in bone of the paranasal sinuses ${ }^{(1)}$. Osteitis is thought to be a disordered inflammatory process rather than chronic bone infection or osteomyelitis, similar to recent research into the mucosal inflammation of CRS ${ }^{(5)}$.

Eosinophilic chronic rhinosinusitis (ECRS) is a subtype of recalcitrant CRS. Patients with ECRS having tissue eosinophil greater than 10 cells per high power field (HPF) have worse disease severity ${ }^{(6)}$ and poorer treatment outcomes compared to noneosinophilic CRS ${ }^{(7)}$. A recent study by Mehta et al. ${ }^{\left({ }^{(8)}\right.}$ found a correlation between osteitis of the paranasal sinuses, based on radiology, with serum and sputum eosinophil levels in asthmatic patients. Additionally, Tran et al., ${ }^{(9)}$ found that patients with CRS with eosinophilic mucin had an increased rate of restenosis and revision surgery after endoscopic modified Lothrop procedure. These findings suggest that tissue eosinophila and a systemic response with serum eosinophilia may predispose or be associated to the osteitic bone seen in recalcitrant CRS. The objective of this study is to investigate an association between osteitis and eosinophilic markers of ECRS.

\section{Material and methods}

Study design

A cross-sectional study of consecutive patients undergoing sinus surgery was undertaken. The study had ethical approval from the St Vincent's Hospital institutional review board.

\section{Patient population}

Adult patients ( $>18$ years) with CRS with or without polyps who underwent ESS in a tertiary referral clinic were reviewed. CRS patients were defined according to EPOS ${ }^{(10)}$. All patients underwent ESS after failing previous medical therapy. No patients used oral steroid for 4 weeks prior to surgery.

Demographic data was recorded. Comorbidity of asthma was defined as clinically using an inhaled $\beta$-agonist or corticosteroid. Patients with suspected aspirin sensitivity on history were confirmed with a nasal lysine aspirin challenge as per the European Guidelines ${ }^{(11)}$. The Sino-Nasal Outcome Test 22 (SNOT-22) was used for disease-specific quality of life assessment ${ }^{(12)}$ Preoperative Lund-Kennedy endoscopy scores were recorded. A structured histopathology report was used to define inflammatory features of the disease ${ }^{(6)}$. Histopathology reported tissue eosinophilia ( $<5$ per high power field (HPF), 5 - 10 per HPF, > 10 per HPF), Charcot-Leyden Crystals (absent, present), eosinophil aggregates (absent, present) and severity of inflammation (absent, mild, moderate and severe). Three high power fields were analyzed to reach a consensus as the density of eosinophilia. The seromarkers reported were: eosinophil count $\left(\mathrm{x} 10^{9} / \mathrm{L}\right)$, total $\operatorname{lgE}(\mathrm{kU} / \mathrm{L}), \mathrm{C}$-reactive protein $(\mathrm{CRP})(\mathrm{mg} / \mathrm{L})$ and erythrocyte sedimentation rate (ESR) ( $\mathrm{mm} /$ hour). All preoperative computed tomography $(\mathrm{CT})$ scans were evaluated with
Lund-Mackay scores.

\section{Global Osteitis Scoring Scale}

Osteitis was defined as the process of bone thickening in patients with CRS was presented ${ }^{(1)}$. Neither distinction is made between osteitis and neo-osteogenesis, nor an assumption of the origins. Bony walls of the paranasal sinuses were assessed for presence, severity and extent of osteitis by using Global Osteitis Score ${ }^{(2)}$. All ten sinuses right and left frontal, anterior ethmoid, posterior ethmoid, maxillary and sphenoid) were scored ranging from 0 to 4 as follows:

0 : Less than $50 \%$ of the sinus walls involved and osteitis $<3$ mm wide (Figure $1 \mathrm{~A}$ ).

1: Less than $50 \%$ of the sinus was involved and $3-5 \mathrm{~mm}$ width (Figure 1B).

2: Less than $50 \%$ of the sinus involved and wider than $5 \mathrm{~mm}$ or greater than $50 \%$ of the sinus wall involved and $<3 \mathrm{~mm}$ wide osteitic changes (Figure 1C).

3: Greater than $50 \%$ of the sinus wall involved and $3-5 \mathrm{~mm}$ (Figure 1D)

4: Greater than $50 \%$ of the sinus wall and thicker than $5 \mathrm{~mm}$ (Figure 1E, 1F).

The Global Osteitis Score ranges from 0 to 40. Woven bone with thickened, irregular, heterogeneous lining of the sinus walls was measured other than normal lamellar bony wall.

\section{Statistical analyses}

Descriptive data was presented as percentage, mean and standard deviation (SD) for parametric data, median and interquartile range (IQR) for non-parametric data. Student's T-test and Mann-Whitney U-test (two-tailed) were used for comparisons of unrelated groups of parametric and non-parametric data, respectively. Kruskal-Wallis was used for comparisons of non-parametric data of more than two groups. Chi squared analysis was used for relationships of nominal variables. Pearson correlation coefficients were performed for linear relationship of scale variables. Spearman's correlation coefficients were used for ordinal values. Statistical analyses were performed using SPSS v 17.0 (Statistical Package for the Social Sciences, Chicago, IL, USA).

\section{Results}

Patient population

Eighty-eight patients with a mean age of $50.3 \pm 13.6$ years were assessed. Forty (45.5\%) patients were female. Nine (10.2\%) patients were smokers and twenty (22.7\%) had asthma. Three (3.4\%) patients had aspirin hypersensitivity. Forty-two (47.7\%) patients were diagnosed as CRSsNP. Thirtythree $(37.5 \%)$ had revision surgery. The number of previous surgery ranged from 1 to 12 . Forty-five (51.1\%) of total patients had some form of osteitis. The prevalence of osteitis was $75.8 \%$ (25/33) for patients with revision surgery and $36.4 \%$ (20/55) for patients with primary surgery. It is acknowledged that the 


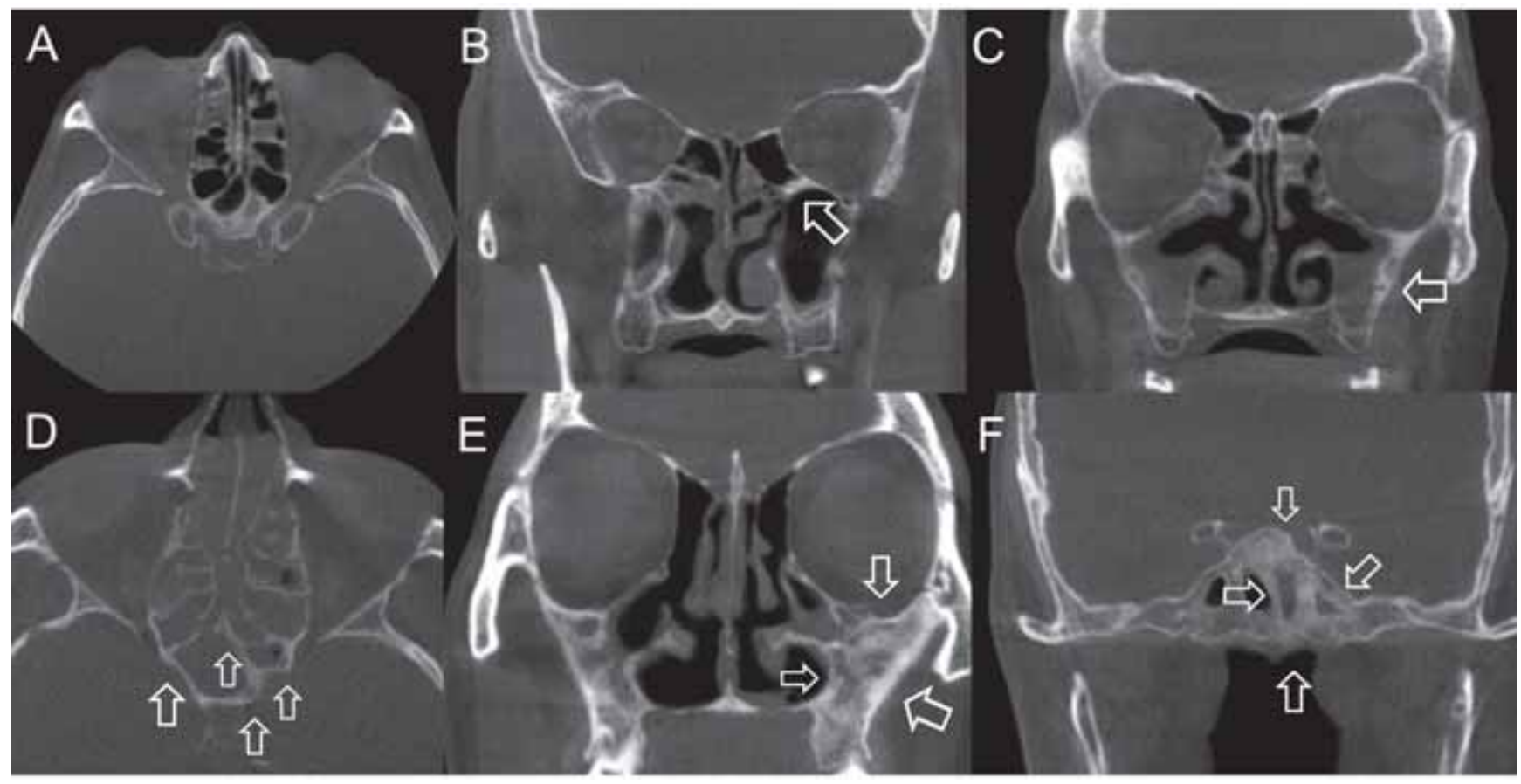

Figure 1. Examples of osteitis (arrow) and Global Osteitis Scoring Scale in CRS patients: (A) score 0 (B) score 1 (C) score 2 (D) score 3 (E) score 4 of maxillary sinus (F) score 4 of sphenoid sinus.

patient population is from a tertiary hospital clinic.

\section{Association between osteitis and clinical presentation}

The mean age of patients with osteitis $(51.6 \pm 14.5)$ was similar to those without $(47.5 \pm 12.0), p=0.18$. The Global Osteitis Score of patients increased with the increase of age $(r=0.23, p$ $=0.04$ ) (Figure 2 ), but was not correlated to age in the primary surgery group ( $r=0.01, p=0.94)$. The presence of osteitis was not associated with gender $(p=0.66)$, smoking ( $p=0.48)$, the comorbidities of asthma $(p=0.40)$ nor aspirin hypersensitivity $(p=1.0)$. Osteitis was associated with the presence of polyps $\left(X^{2}=4.6, p=0.05\right)$ and previous surgery $\left(X^{2}=12.8, p<0.001\right)$. The median Global Osteitis Score was greater in patients with CRSwNP (4.0 (2.0 - 6.0)) than patients with CRSsNP (0.0 (0.0 5.3), $\mathrm{p}=0.03$ ) (Figure 3 ) and greater in patients undergoing revision surgery $(6.0(3.8-10.3))$ than primary surgery $(0.0$ (0.0 - 3.0), p < 0.001) (Figure 4). The median of number of previous surgeries was higher in patients with osteitis (1.0 (0.0 - 2.0)) than those without $(0.0(0.0-0.0), p=0.001)$.

When un-operated CRS patients (primary surgery subgroup) were assessed independently, the Global Osteitis Score of patients with osteitis was neither correlated with age $(p=$ $0.94)$ nor the presence of polyps $(p=0.15)$. The median Global Osteitis Score in patients with CRSwNP (2.5 (1.3 - 4.0)) was not different from patients with CRSsNP $(0.0(0.0-0.0), p=0.23)$. The presence of osteitis was not associated with gender $(\mathrm{p}=$
$0.77)$, smoking $(p=1.0)$, the comorbidities of asthma $(p=0.68)$ and aspirin hypersensitivity $(p=0.43)$.

\section{Association between osteitis and disease severity}

The mean SNOT-22 in patients with osteitis $(2.0 \pm 1.0)$ was not different from those without $(1.9 \pm 1.1), p=0.56$. Patients with osteitis had greater endoscopy score $(6.1 \pm 2.9$ versus $4.4 \pm 3.6$, $\mathrm{p}=0.03)$ and CT score $(14.0 \pm 6.0$ versus $10.1 \pm 5.7, \mathrm{p}=0.005)$ than those without. Data are displayed in Table 1.

Similar findings were found in the primary surgery subgroup. The mean SNOT-22 in patients with osteitis $(1.8 \pm 0.8)$ was not different from those without $(1.7 \pm 1.0), p=0.75$. Patients with osteitis had greater endoscopy score $(6.6 \pm 2.9$ versus $4.5 \pm 3.6$, $\mathrm{p}=0.03)$ and CT score $(15.6 \pm 5.5$ versus $10.4 \pm 5.2, \mathrm{p}=0.002)$ than those without.

\section{Association between osteitis and histopathology}

The median Global Osteitis Score was greater in patients with tissue eosinophilia ( $\geq 10 / \mathrm{HPF})(4.0(1.0-6.0))$ than those with less tissue eosinophil (1.0 (0.0-5.8), $\mathrm{p}=0.04)$ (Figure 5). No association with Charcot-Leyden crystals (4.0 (0.0 - 6.0) versus 2.0 $(0.0-6.0), p=0.65)$ and eosinophil aggregates (4.0 (0.0 - 6.0) versus $2.0(0.0-7.0), p=1.0)$ were found. Data are displayed in Table 2.

Similar findings were made in the primary surgery subgroup. 


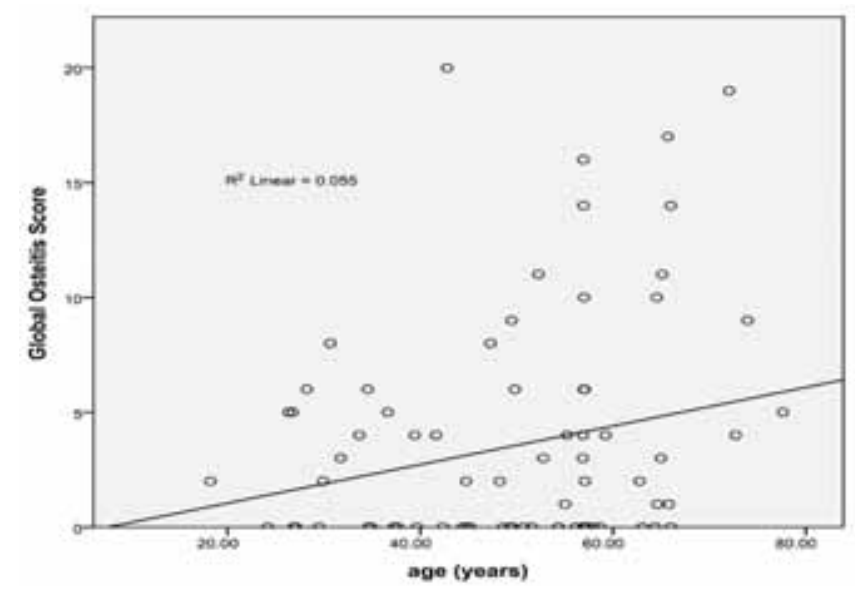

Figure 2. Global Osteitis Score by age.

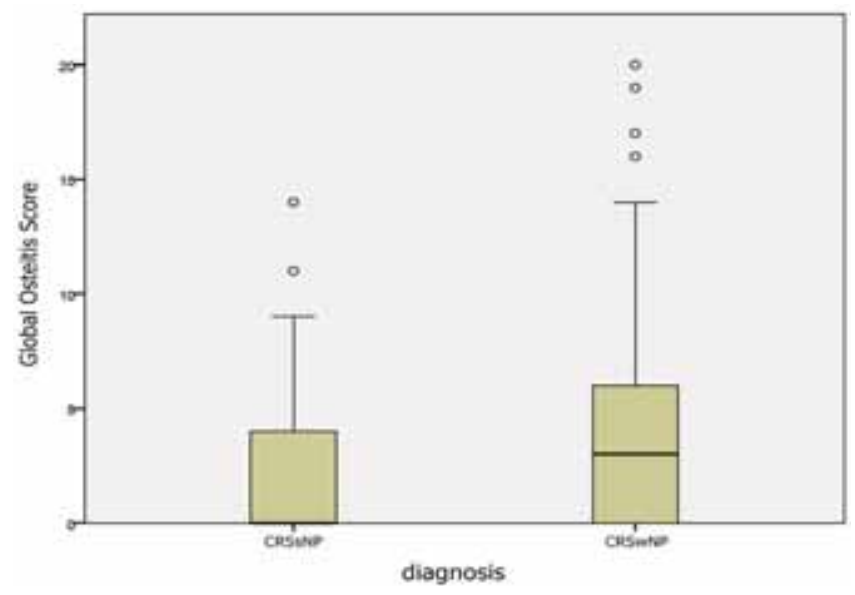

Figure 3. Global Osteitis Score by diagnosis.
The median Global Osteitis Score was greater in patients with tissue eosinophilia ( $\geq 10 / \mathrm{HPF})(3.0(0.0-4.0)$ ) than those with less tissue eosinophilia (0.0 (0.0 - 0.0), $\mathrm{p}=0.001)$ (Figure 5). There was no significant difference $(p=0.27)$ between the median Global Osteitis Score for the primary group and the severity of tissue inflammation (Kruskal Wallis). The Global Osteitis Score was not correlated to age in the primary surgery group $(r=0.01, p=0.94)$.

\section{Association between osteitis and seromarkers}

Data are displayed in Table 1. Patients with osteitis had higher median serum eosinophil count $\left(0.3(0.2-0.5) \times 10^{9} / \mathrm{L}\right.$ versus $\left.0.1(0.1-0.2) \times 10^{9} / \mathrm{L}, \mathrm{p}<0.001\right)$ and median serum IgE level (78.0 (29.5 - 252.0) versus $29.0(18.8-69.3), p=0.01)$ than those without. Median of CRP (1.9 (0.8 - 2.5) versus $2.5(0.9-7.0), p$ $=0.20)$ and $\operatorname{ESR}(6.0(3.0-12.5)$ versus $5.5(2.0-10.0), \mathrm{p}=0.40)$ were not different between patients with osteitis and without. The median Global Osteitis Score was greater in patients with high serum eosinophilia $\left(\geq 0.3 \times 10^{9} / \mathrm{L}\right)(5.0(1.8-11.0)$ versus $0.0(0.0-4.0), p=0.004$ ) (Figure 6).

In the primary surgery subgroup, patients with osteitis had higher median serum eosinophil count $(0.3(0.1-0.6) \times 10 \% / L$ versus $\left.0.1(0.1-0.2) \times 10^{9} / \mathrm{L}, \mathrm{p}=0.01\right)$ compared to patients without osteitis but similar median serum IgE level (41.0 (25.0 - 63.0) versus 31.0 (19.0 - 73.0), $p=0.46)$. The CRP (1.4 (0.8 - 2.2) versus $2.1(0.6-5.2), \mathrm{p}=0.45)$ and $\operatorname{ESR}(5.0(2.0-12.3)$ versus $5.0(2.0-10.0), p=0.73$ ) were not different between groups. The median Global Osteitis Score was greater in patients whose serum eosinophilia was beyond previously reported threshold $(6,13)\left(\geq 0.3 \times 10^{9} / \mathrm{L}\right)(3.0(0.8-6.5)$ versus $0.0(0.0$ $0.0), p=0.002$ ) (Figure 6).

\section{Discussion}

The prevalence of osteitis in this study was similar to Georgalas et al., (2) and Lee et al. ${ }^{(3)}$. The presence of osteitic bones is widely accepted to be associated with previous surgery and it is also shown by this study. However, osteitis is multifactorial and can be found in non-operated patients. It is associated with other features of ECRS such as the presence of polyps, high tissue eosinophils $\geq 10 / \mathrm{HPF}$, high serum eosinophils $\geq 0.3$ $\mathrm{x} 10^{\circ} / \mathrm{L}$ and high serum IgE. Importantly, in a primary surgery subgroup, only true eosinophilic states, which are tissue and serum eosinophilia show significant correlations. Although nasal polyps is a clinical feature of ECRS, the phenotype and the endotype are different in many patients, as tissue eosinophilia is also present in up to $19 \%$ of patients with CRSsNP ${ }^{(6)}$.

The relationship between osteitis of paranasal sinuses and high eosinophil level in serum and sputum was also reported by Mehta et al. ${ }^{(8)}$. The pathogenesis of this relationship is not known. To date bacteria have not been demonstrated as common feature in the bone of CRS patients ${ }^{(1)}$. The pathogenesis of osteitis may be either infection as shown from animal models studies ${ }^{(14)}$ and/or inflammation. A chronic process of eosinophilic mucosal inflammation of ECRS may initiate bone remodeling including periosteal reaction, osteoclast proliferation, bone resorption, new bone formation, fibrosis and cellular infiltration. This response is similar to the reaction to other stimuli such as mechanical stress and post-traumatic repair ${ }^{(1)}$. We do not have sufficient information to determine the length of symptoms (beyond 3 months) for our patient population. The osteitis may be related to duration of inflammation. To explore this further we looked at the relationship of age and osteitis score. There was no correlation between the age of the patients and osteitis in the non-operated subgroup $(r=0.01$, $p=0.94)$. There is an association with age and the total group 


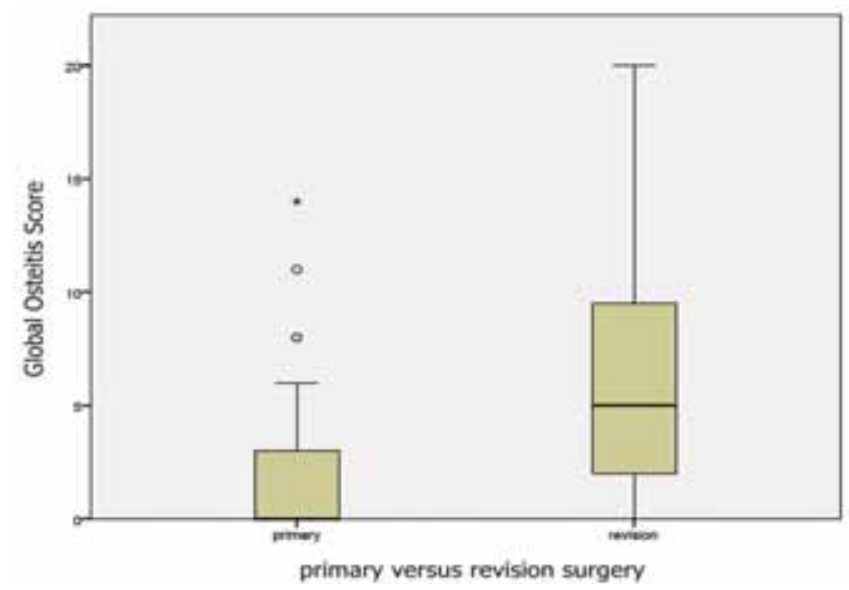

Figure 4. Global Osteitis Score by primary versus revision surgery.

but this is confounded by prior surgery in the total population. Although eosinophilia is associated with clinical severity, the histological severity of inflammation was not associated with and global osteitis score was found in the primary surgery subgroup ( $p=0.27$ ). Possible alternative explanations of this association with eosinophilia could include a chronic inflammation dominated by a cytokine milieu of Th2 inflammation affecting not only sinus mucosa but also the bony walls. The systemic release of pro-inflammatory cytokines, such as IL-4 an known inducer of neo-osteogenesis ${ }^{(15)}$, may trigger bone neo-osteogenesis in significant ECRS cases. However eosinophil infiltration is not common in bony histopathology of CRS patient and a systemic response may be required to initiate the osteitis. The majority of current animal models of CRS are based on ostial obstruction and bacterial inoculation ${ }^{(14)}$ and not an eosinophilic airway process and thus may be unreliable surrogates for research on bone changes.

Patients with osteitis have greater disease severity in endoscopy and CT score but similar symptoms compared to those without. These were similarly reported by other studies ${ }^{(3,16-18)}$. The greater disease severity in patients with ECRS shown in our previous study is analogous to these findings ${ }^{(6)}$. It is also shown in the literature that CRS patients with osteitis have poorer treatment outcomes ${ }^{(19)}$. The ECRS subgroup is also related to an increased rate of restenosis and revision surgery after endoscopic sinus surgery ${ }^{(7)}$ and modified Lothrop procedure (9). If there is a causal link between osteitis, strong tissue eosi-
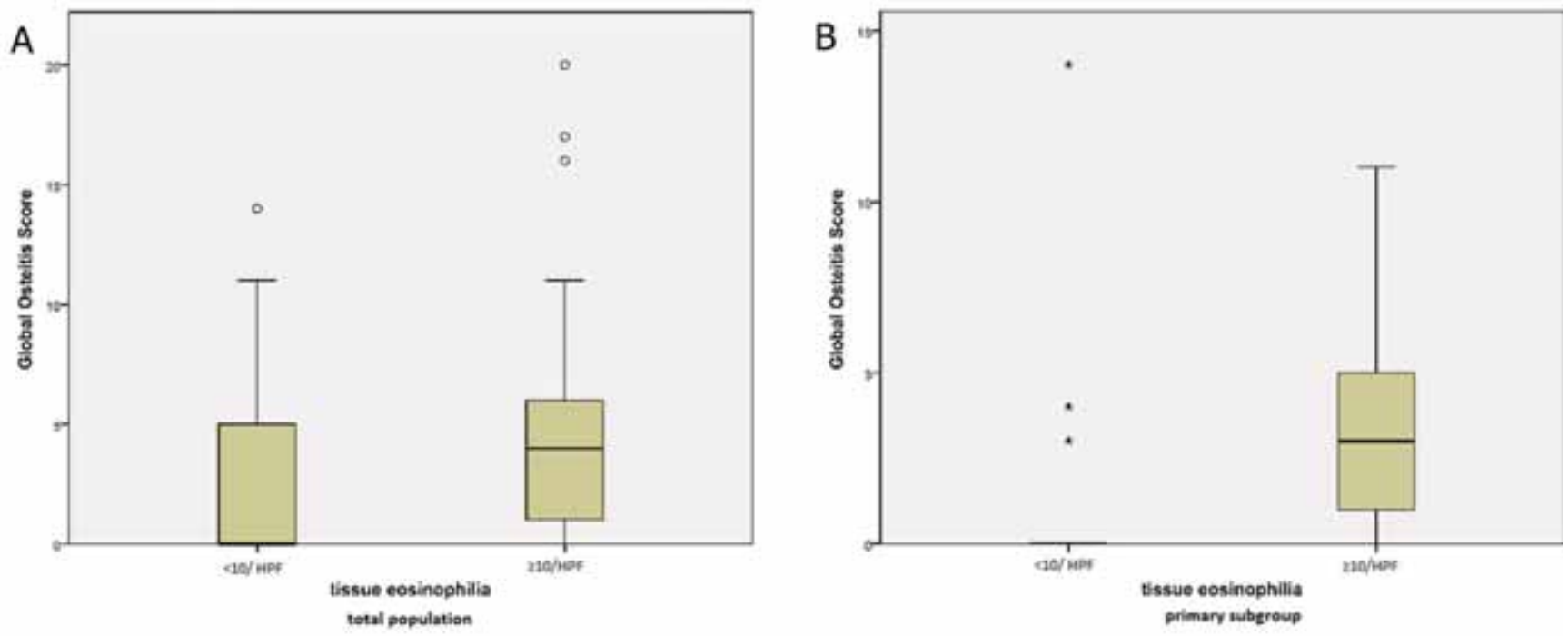

Figure 5. Global Osteitis Score by tissue eosinophilia ( $\geq 10 / \mathrm{HPF}$ ) (A) total population (B) primary subgroup. 
Table 1. Disease severity and seromarkers by the presence of osteitis in total population and primary surgery subgroup.

\begin{tabular}{|c|c|c|c|c|c|c|c|}
\hline & & \multicolumn{3}{|c|}{ Total population } & \multicolumn{3}{|c|}{ Primary surgery subgroup } \\
\hline & & Non-osteitis & Osteitis & p-value & Non-osteitis & Osteitis & p-value \\
\hline \multirow{3}{*}{$\begin{array}{l}\text { Disease severity } \\
\quad(\text { mean } \pm \text { SD) }\end{array}$} & SNOT-22 & $1.85 \pm 1.1$ & $2.03 \pm 1.0$ & 0.56 & $1.7 \pm 1.0$ & $1.8 \pm 0.8$ & 0.75 \\
\hline & $\begin{array}{l}\text { Endoscopy } \\
\text { score }\end{array}$ & $4.38 \pm 3.6$ & $6.07 \pm 2.9$ & 0.03 & $4.5 \pm 3.6$ & $6.6 \pm 2.9$ & 0.03 \\
\hline & CT score & $10.14 \pm 5.7$ & $13.95 \pm 6.0$ & 0.005 & $10.4 \pm 5.2$ & $15.6 \pm 5.5$ & 0.002 \\
\hline \multirow{4}{*}{$\begin{array}{l}\text { Seromarkers } \\
\text { (median (IQR)) }\end{array}$} & $\begin{array}{l}\text { Serum } \\
\text { eosinophil } \\
\left(x 10^{9} / L\right)\end{array}$ & $0.1(0.1-0.2)$ & $0.3(0.2-0.5)$ & $<0.001$ & $0.1(0.1-0.2)$ & $0.3(0.1-0.6)$ & 0.01 \\
\hline & $\begin{array}{l}\text { Serum IgE } \\
\text { (kU/L) }\end{array}$ & $29.0(18.8-69.3)$ & $\begin{array}{c}78.0 \\
(29.5-252.0)\end{array}$ & 0.01 & $31.0(19.0-73.0)$ & $41.0(25.0-63.0)$ & 0.46 \\
\hline & $\mathrm{CRP}(\mathrm{mg} / \mathrm{L})$ & $2.5(0.9-7.0)$ & $1.9(0.8-2.5)$ & 0.20 & $2.1(0.6-5.2)$ & $1.4(0.8-2.2)$ & 0.45 \\
\hline & ESR (mm/hour) & $5.5(2.0-10.0)$ & $6.0(3.0-12.5)$ & 0.40 & $5.0(2.0-10.0)$ & $5.0(2.0-12.3)$ & 0.73 \\
\hline
\end{tabular}

Table 2. Global Osteitis Score by histopathology in total population and primary surgery subgroup.

\begin{tabular}{|c|c|c|c|c|c|}
\hline \multicolumn{2}{|c|}{ Factors } & \multicolumn{2}{|c|}{ Global Osteitis Score in total population } & \multicolumn{2}{|c|}{$\begin{array}{c}\text { Global Osteitis Score in primary } \\
\text { surgery subgroup }\end{array}$} \\
\hline & & Median (IQR) & p-value & Median (IQR) & p-value \\
\hline \multirow{2}{*}{$\begin{array}{l}\text { Tissue } \\
\text { eosinophilia }\end{array}$} & $<10 / \mathrm{HPF}$ & $1.0(0.0-5.8)$ & 0.04 & $0.0(0.0-0.0)$ & 0.001 \\
\hline & $\geq 10 / \mathrm{HPF}$ & $4.0(1.0-6.0)$ & & $3.0(0.0-4.0)$ & \\
\hline \multirow[t]{2}{*}{ Charcot-Leyden } & absent & $2.0(0.0-6.0)$ & 0.65 & $0.0(0.0-2.0)$ & 0.98 \\
\hline & present & $4.0(0.0-6.0)$ & & $2.0(0.0-5.5)$ & \\
\hline \multirow{3}{*}{$\begin{array}{l}\text { Eosinophil } \\
\text { aggregates }\end{array}$} & absent & $2.0(0.0-7.0)$ & 1.0 & $0.0(0.0-2.3)$ & 0.68 \\
\hline & present & $4.0(0.0-6.0)$ & & $0.0(0.0-5.0)$ & \\
\hline & absent & NA & 0.03 & NA & 0.27 \\
\hline \multirow{3}{*}{$\begin{array}{l}\text { Severity of } \\
\text { inflammation }\end{array}$} & mild & $0.0(0.0-4.0)$ & & $0.0(0.0-2.8)$ & \\
\hline & moderate & $3.0(0.0-5.8)$ & & $1.0(0.0-4.0)$ & \\
\hline & severe & $6.0(3.5-13.5)$ & & $4.5(0.8-9.8)$ & \\
\hline
\end{tabular}

NA: There were no patients with absent inflammation.
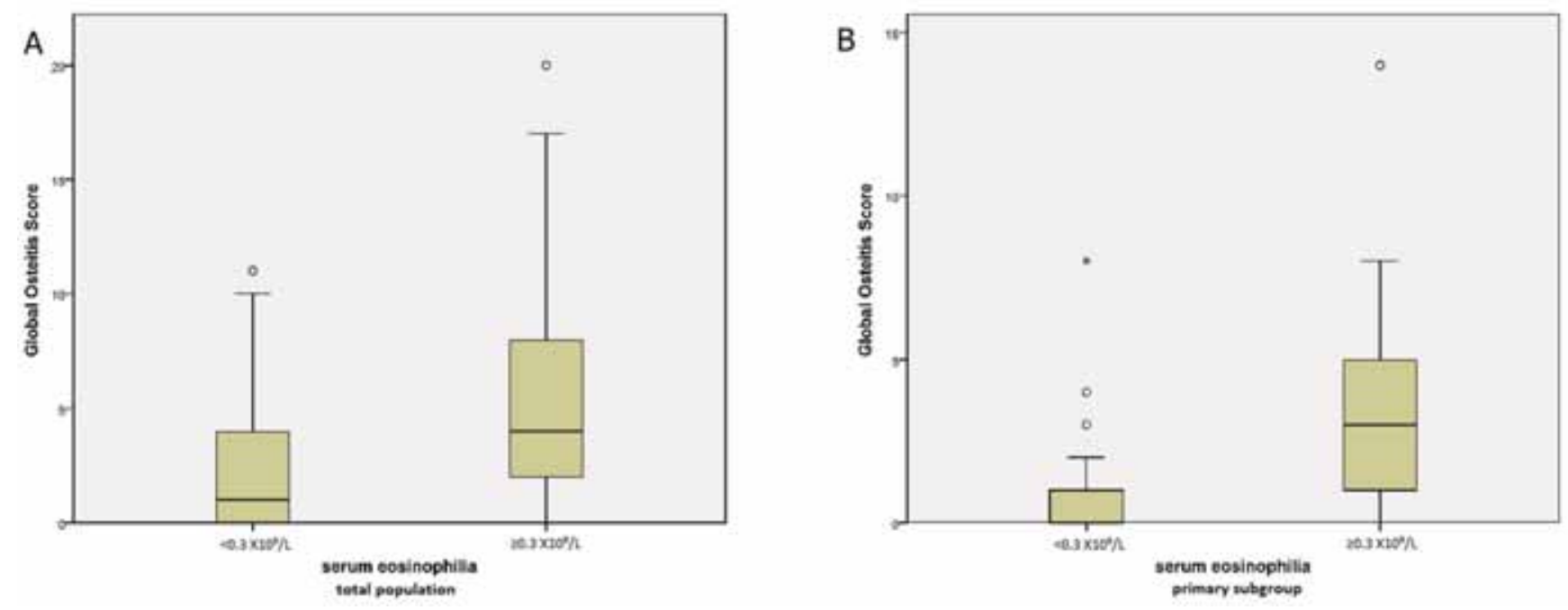

Figure 6. Global Osteitis Score by serum eosinophilia ( $\geq 0.3 \times 109 / \mathrm{L})$ (A) total population (B) primary subgroup. 
nophilia and serum eosinophilia then structured histopathology ${ }^{(6)}$ reporting and serum analysis may be helpful to define a subgroup at risk for osteitis. The simple concept of endoscopic sinus surgery to create ventilation and drainage may be an inappropriate philosophy for patients with osteitis. Remnants of diseased bone may serve as a constant nidus for inflammation, inducing recurrent edema and hypertrophy of the overlying mucosa ${ }^{(1)}$ and complete removal may be required. Additionally, a strong post-operative corticosteroid therapy regime may be appropriate for the patients with serum eosinophilia $>0.3$ $x 10^{9} / \mathrm{L}$ and high tissue eosinophilia (> 10/HPF) to prevent postsurgery osteitis if systemic mediators are involved in osteitis formation.

\section{Conclusion}

Osteitis is associated with tissue and serum eosinophilia in both patients with and without prior surgery. Potentially, patients with these features may benefit the most from post- operative corticosteroid therapy to prevent further osteitis and should be an area of future research.

\section{Author Contribution}

KS: data collection, analysis and manuscript preparation; RM: data collection; DC: manuscript review; EP: data collection and analysis; RS: Manuscript review and assessment; PE: pathology assessment and review; RJH: study concept, data analysis, manuscript review.

\section{Conflict of interest}

Richard J. Harvey has served on an advisory board for Schering Plough and Glaxo-Smith-Kline, previous consultant with Medtronic and Olympus, speakers bureau for Merck Sharp Dohme and Arthrocare and has received grant support from NeliMed. Raymond Sacks is consultant for Medtronic and Nycomed, speakers bureau for Merck Sharp Dohme and Arthrocare.

\section{References}

1. Videler WJ, Georgalas C, Menger DJ, Freling NJ, van Drunen CM, Fokkens WJ. Osteitic bone in recalcitrant chronic rhinosinusitis. Rhinology. 2011; 49: 139-147.

2. Georgalas C, Videler W, Freling N, Fokkens WJ. Global Osteitis Scoring Scale and chronic rhinosinusitis: a marker of revision surgery. Clin Otolaryngol. 2010; 35: 455-461.

3. Lee JT, Kennedy DW, Palmer JN, Feldman M, Chiu AG. The incidence of concurrent osteitis in patients with chronic rhinosinusitis: a clinicopathological study. Am J Rhinol. 2006; 20: 278-282.

4. Kennedy DW, Senior BA, Gannon FH, Montone KT, Hwang P, Lanza DC. Histology and histomorphometry of ethmoid bone in chronic rhinosinusitis. Laryngoscope. 1998; 1084 Pt 1: 502-507.

5. Kern RC, Conley DB, Walsh W, et al. Perspectives on the etiology of chronic rhinosinusitis: an immune barrier hypothesis. Am J Rhinol. 2008; 22: 549-559.

6. Snidvongs K, Lam M, Sacks R, et al. Structured histopathology profiling of chronic rhinosinusitis in routine practice. Int Forum Allergy Rhinol. 2012; doi: 10.1002/ alr.21032. [Epub ahead of print].

7. Soler ZM, Sauer D, Mace J, Smith TL. Impact of mucosal eosinophilia and nasal polyposis on quality-of-life outcomes after sinus surgery. Otolaryngol Head Neck Surg. 2010; 142: 64-71.
8. Mehta V, Campeau NG, Kita H, Hagan JB. Blood and sputum eosinophil levels in asthma and their relationship to sinus computed tomographic findings. Mayo Clin Proc. 2008; 83: 671-678

9. Tran KN, Beule AG, Singal D, Wormald PJ. Frontal ostium restenosis after the endoscopic modified Lothrop procedure. Laryngoscope. 2007;117: 1457-1462.

10. Fokkens W, Lund V, Mullol J et al. European position paper on rhinosinusitis and nasal polyps 2012. Rhinol Suppl. 2012: 1-296.

11. Nizankowska-Mogilnicka E, Bochenek G, Mastalerz L, et al. EAACI/GA2LEN guideline: aspirin provocation tests for diagnosis of aspirin hypersensitivity. Allergy. 2007; 62: 1111-1118.

12. Hopkins C, Gillett S, Slack R, Lund VJ, Browne JP. Psychometric validity of the 22-item Sinonasal Outcome Test. Clin Otolaryngol. 2009; 34: 447-454

13. Han DH, Kim SW, Cho SH, et al. Predictors of bronchial hyperresponsiveness in chronic rhinosinusitis with nasal polyp. Allergy. 2009; 64: 118-122.

14. Perloff JR, Gannon FH, Bolger WE, Montone KT, Orlandi R, Kennedy DW. Bone involvement in sinusitis: an apparent pathway for the spread of disease. Laryngoscope. 2000; 110: 2095-2099.

15. Lorenzo JA. The role of cytokines in the regulation of local bone resorption. Crit Rev Immunol. 1991; 11: 195-213.
16. Giacchi RJ, Lebowitz RA, Yee HT, Light JP, Jacobs JB. Histopathologic evaluation of the ethmoid bone in chronic sinusitis. Am J Rhinol. 2001; 15: 193-197.

17. Bhandarkar ND, Mace JC, Smith TL. The Impact of Osteitis on Disease Severity Measures and Quality of Life Outcomes in Chronic Rhinosinusitis. Int Forum Allergy Rhinol. 2011; 1: 372-378.

18. Cho SH, Min HJ, Han HX, Paik SS, Kim KR. CT analysis and histopathology of bone remodeling in patients with chronic rhinosinusitis. Otolaryngol Head Neck Surg. 2006; 135: 404-408.

19. Kim HY, Dhong HJ, Lee HJ, et al. Hyperostosis may affect prognosis after primary endoscopic sinus surgery for chronic rhinosinusitis. Otolaryngol Head Neck Surg. 2006; 135: 94-109.

Kornkiat Snidvongs

The Australian School of Advanced

Medicine

2 Technology Place

Macquarie University NSW 2109

Australia 